\title{
Regulation of multiple target genes by miR-1 and miR-206 is pivotal for C2C12 myoblast differentiation
}

\author{
Katarzyna Goljanek-Whysall ${ }^{1}$, Helio Pais ${ }^{2}$, Tina Rathjen ${ }^{3}$, Dylan Sweetman ${ }^{1, \star}$, Tamas Dalmay $^{3}$ \\ and Andrea Münsterberg ${ }^{1, \pm}$ \\ ${ }^{1}$ Cell and Developmental Biology, School of Biological Sciences, University of East Anglia, Norwich Research Park, Norwich, NR4 7TJ, UK \\ ${ }^{2}$ Computing Sciences, University of East Anglia, Norwich Research Park, Norwich, NR4 7TJ, UK \\ ${ }^{3}$ Molecular Biology, School of Biological Sciences, University of East Anglia, Norwich Research Park, Norwich, NR4 7TJ, UK \\ *Present address: Division of Animal Sciences, School of Biosciences, University of Nottingham, Sutton Bonington Campus, Loughborough, Leicestershire LE12 5RD, UK \\ ${ }^{\ddagger}$ Author for correspondence (a.munsterberg@uea.ac.uk) \\ Accepted 31 March 2012 \\ Journal of Cell Science 125, 3590-3600 \\ (C) 2012. Published by The Company of Biologists Ltd \\ doi: $10.1242 / j c s .101758$
}

\section{Summary}

MicroRNAs are short non-coding RNAs involved in post-transcriptional regulation of multiple messenger RNA targets. The miR-1/ miR-206 family is expressed during skeletal muscle differentiation and is an integral component of myogenesis. To better understand miR-1/miR-206 function during myoblast differentiation we identified novel target mRNAs by microarray and characterized their function in $\mathrm{C} 2 \mathrm{C} 12$ myoblasts. Candidate targets from the screen were experimentally validated together with target genes that were predicted by three different algorithms. Some targets characterised have a known function in skeletal muscle development and/or differentiation and include Meox2, RARB, Fzd7, MAP4K3, CLCN3 and NFAT5, others are potentially novel regulators of myogenesis, such as the chromatin remodelling factors Smarcd2 and Smarcb1 or the anti-apoptotic protein SH3BGRL3. The expression profiles of confirmed target genes were examined during $\mathrm{C} 2 \mathrm{C} 12$ cell myogenesis. We found that inhibition of endogenous miR-1 and miR-206 by antimiRs blocked the downregulation of most targets in differentiating cells, thus indicating that microRNA activity and target interaction is required for muscle differentiation. Finally, we show that sustained expression of validated miR-1 and/or miR-206 targets resulted in increased proliferation and inhibition of $\mathrm{C} 2 \mathrm{C} 12$ cell myogenesis. In many cases the expression of genes related to non-muscle cell fates, such as chondrogenesis, was activated. This indicates that the concerted downregulation of multiple microRNA targets is not only crucial to the skeletal muscle differentiation program but also serves to prevent alternative cell fate choices.

Key words: miR-1, miR-206, Skeletal muscle, C2C12 myoblast, MicroRNA, Cell fate

\section{Introduction}

MicroRNAs (miRNAs or miRs) are short non-coding RNAs, found in both plants and animals. In animals, miRNAs bind with imperfect complementarity to target sites that are typically found in the $3^{\prime}$ UTR of messenger RNAs. This results in inhibition of translation and in many cases transcript degradation (Carthew, 2006; Guo et al., 2010). Post-transcriptional regulation of gene expression by miRNAs is an important feature of cell differentiation programs and it has been predicted that there are many as yet undiscovered miRNAs in the genomes of human and other higher vertebrates (Bartel, 2004). Although some miRNAs have been characterised in detail, in most cases there is only limited information about their function. This is in part due to the incomplete complementarity of miRNAs with their targets, which makes the identification of biologically relevant targets more challenging (Bartel, 2009). Different algorithms predict numerous potential targets, not all of which are genuinely affected by miRNAs (Krützfeldt et al., 2006).

$\mathrm{C} 2 \mathrm{C} 12$ myoblast cells are widely used as a model for skeletal muscle cell differentiation. Undifferentiated $\mathrm{C} 2 \mathrm{C} 12$ cells express Myf5 and MyoD; however, the ability of these MRFs to induce differentiation is blocked under growth conditions. The removal of growth factors or the downregulation of their receptors leads to myoblast differentiation, which correlates with expression of miR-1 and miR-206, two highly related but not identical
microRNAs, which are conserved across many species (Sweetman et al., 2008). The specific versus redundant functions of miR-1 and miR-206 are currently not well understood. In addition, miR-1 and miR-206 are co-expressed with a member of the miR-133 family, which is generated from the same primary transcript but has a completely different sequence and therefore a distinct set of targets (Chen et al., 2006; Kim et al., 2006; Rao et al., 2006; Sweetman et al., 2008). Experiments in $\mathrm{C} 2 \mathrm{C} 12$ cells suggested that miR-1 and miR-206 promote differentiation of myoblasts through downregulation of HDAC4 and the p180 subunit of DNA polymerase alpha, whilst miR-133 promotes proliferation through downregulation of SRF (Chen et al., 2006; Kim et al., 2006). In addition, it has been shown that miR-1 and miR-133 influence the differentiation behaviour of rhabdomyosarcoma cells and it might be possible to exploit their pro-myogenic functions for therapeutic purposes (Rao et al., 2010).

In vertebrate embryos, myogenic cells derived from embryonic somites give rise to skeletal muscles of the trunk and limbs, and skeletal myogenesis serves as a paradigm for the study of cell fate commitment in response to instructive cues. We, and others, showed that miR-1 and miR-206 expression is restricted to developing skeletal and cardiac muscle during vertebrate embryogenesis (Sweetman et al., 2006; Darnell et al., 2006; Wienholds et al., 2005). In addition, they are regulated by the 
Myogenic Regulatory Factors (MRFs) in vivo (Hinits et al., 2011; Sweetman et al., 2008; Sweetman et al., 2006) consistent with the finding that MyoD and myogenin bind to regions upstream of $m i R-1$ and induce its expression in cell culture (Rao et al., 2006; van Rooij et al., 2008). Recently we demonstrated that miR-1 and miR-206 mediate appropriate timing of myogenesis in developing somites by contributing to the downregulation of Pax3 as committed myoblasts differentiate (Goljanek-Whysall et al., 2011). We have also identified additional microRNAs expressed in embryonic somites indicating that multiple microRNAs are involved in their differentiation (Rathjen et al., 2009). However, the precise roles of many of these miRs and how they act to regulate muscle development and differentiation remain to be identified.

Here we focus on miR-1 and miR-206 and identify novel targets using two different approaches: microarray screening of C2C12 cells transfected with miR-206 or antimiR-206, as well as target predictions by three widely used target prediction algorithms, PicTar (Krek et al., 2005), TargetScanS (Friedman et al., 2009; Grimson et al., 2007; Lewis et al., 2005) and miRanda (Griffiths-Jones et al., 2008). Systematic analyses of predicted targets led to a list of candidates with potential biological relevance. A selection of these was experimentally validated using luciferase reporter assays with $3^{\prime}$ UTR sensor constructs, leading to confirmation or elimination of predicted targets. Confirmed targets were then analysed in vivo, and we showed that endogenous expression levels of these genes are sensitive to miR-206/miR-1 overexpression, and that this miRNA-induced downregulation was inhibited by specific antimiRs. This approach identified CLCN3, Meox2, Smarcd2, Smarcb1, Fzd7, MAP4K3, NFAT5 and RARB as miR-1/miR206 targets during differentiation of the $\mathrm{C} 2 \mathrm{C} 12$ myoblast cell line. The biological relevance of this regulation was confirmed by sustained overexpression of targets, which we show interferes with the myogenic differentiation program of $\mathrm{C} 2 \mathrm{C} 12$ cells, leading to disruption of cell cycle exit, alteration of fibre morphology or activation of genes related to non-myogenic differentiation programs. Thus, this approach identified novel targets for miR-1/miR-206, whose negative regulation is crucial to orchestrate myogenic differentiation of $\mathrm{C} 2 \mathrm{C} 12$ myoblasts.

\section{Results}

\section{Transcript profiling identifies novel potential targets of miR-206}

To identify effects of miR-206 on mRNA levels we generated transcript profiles of $\mathrm{C} 2 \mathrm{C} 12$ cells on day one of myogenic differentiation, when the endogenous level of miR-206 is still low (D1-control). This was compared to $\mathrm{C} 2 \mathrm{C} 12$ cells transfected with miR-206 (D1-miR-206), thus prematurely increasing the activity of miR-206. We also obtained samples on day two of differentiation (D2), when endogenous miR-206 expression is high. Cells were either untreated (D2-control) or transfected with an antimiR that inhibits miR-206 (D2-antimiR) (Fig. 1). Northern analysis showed that antimiR-206 affected the expression of both miR-1 and miR-206 (Fig. 2D).

Microarray analyses were performed on a mouse Illumina array (Cambridge Genomic Services). The data set has been submitted to the GEO database at NCBI (accession number GSE34069). Bioinformatics analysis showed that antimiR transfection affected the expression level of a greater number of genes than transfection with miR-206 (Fig. 1A,B). It is possible that at day one most targets are not yet expressed and therefore increasing miR-206 activity has a limited effect. Furthermore, the expression profile obtained with D2-antimiR
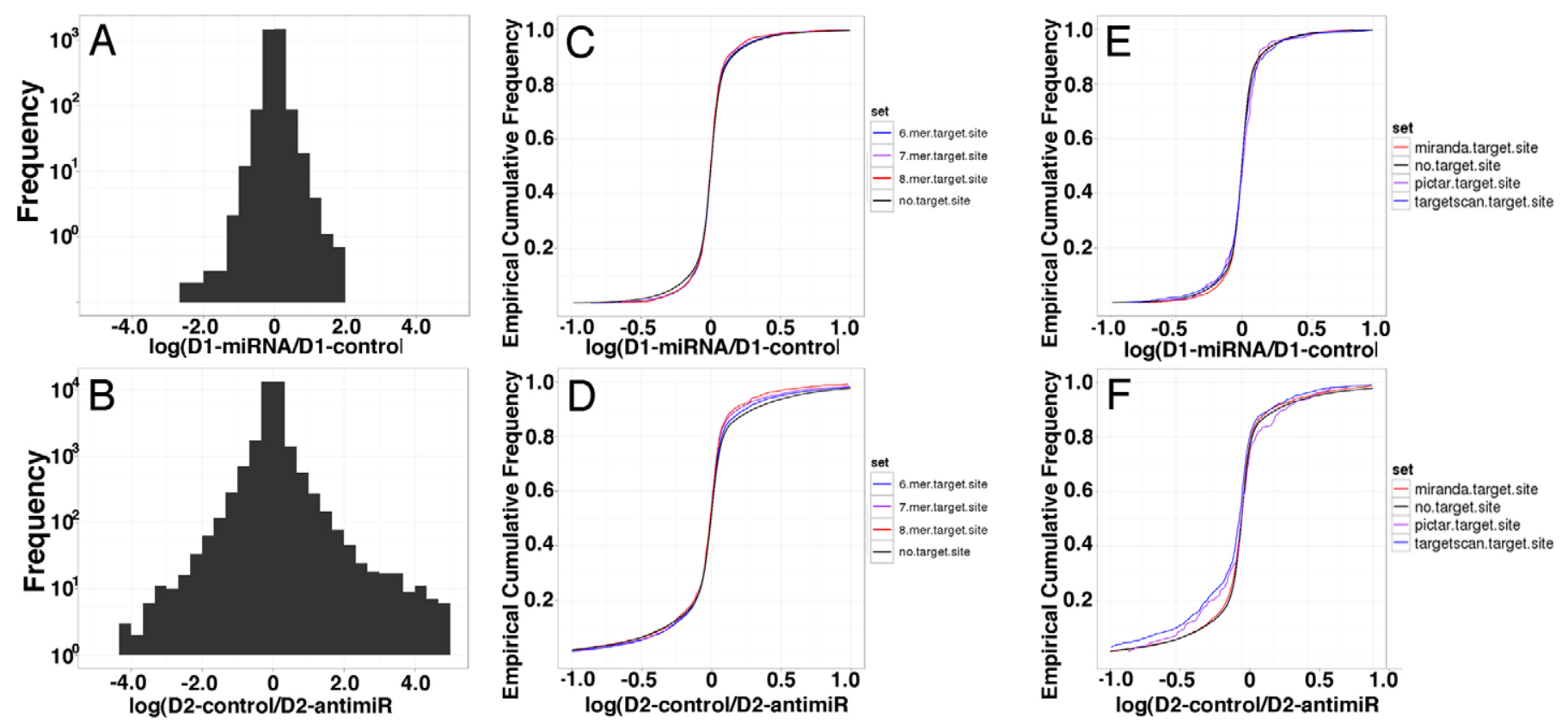

Fig. 1. Mouse Illumina array of C2C12 cells transfected with miR-206 or with antimiR-206. (A,B) Differential expression histograms for pairwise

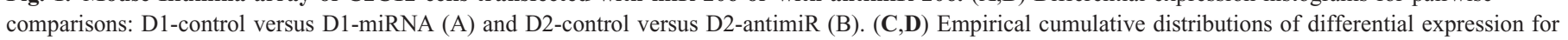

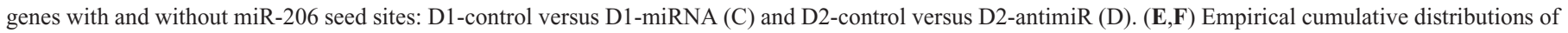

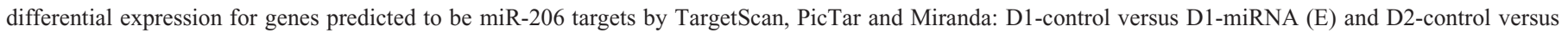
D2-antimiR (F). 


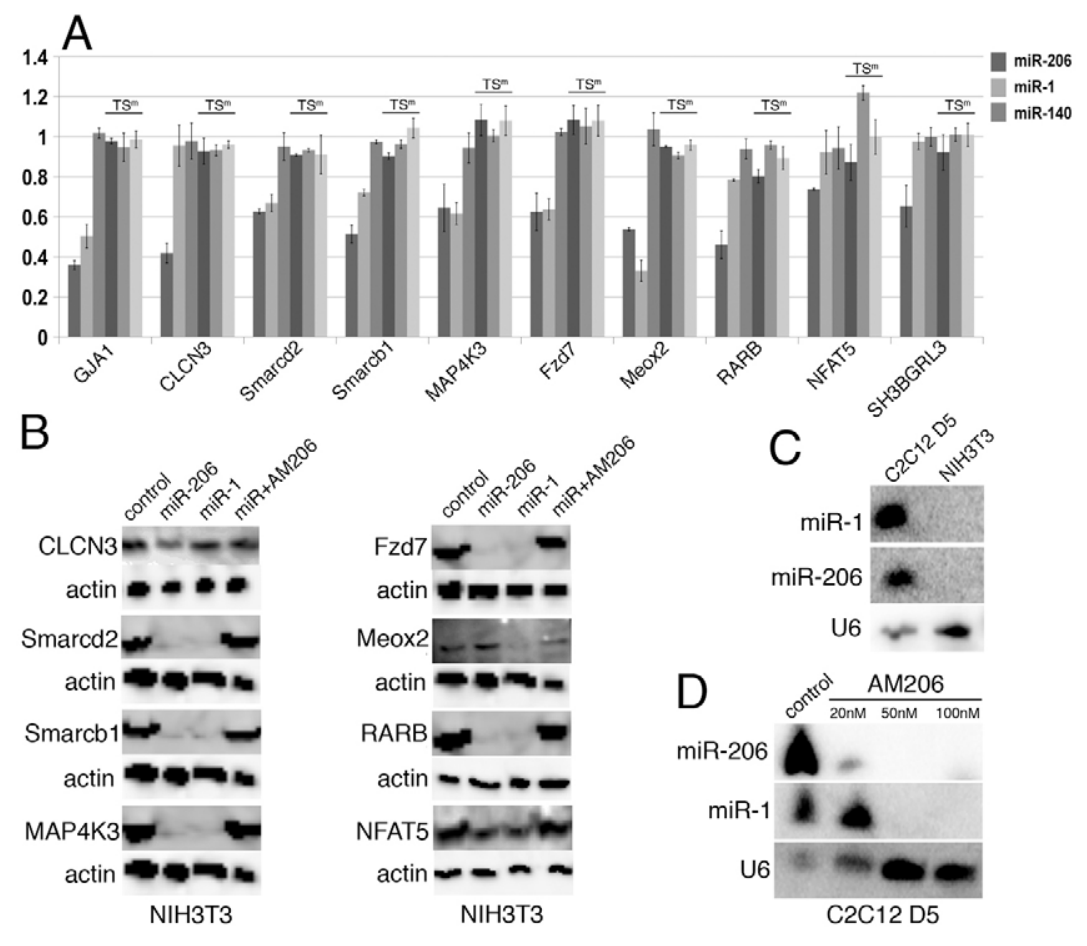

Fig. 2. miR-206 and miR-1 repress candidate target genes through elements in the $\mathbf{3}^{\prime} \mathrm{UTR}$. (A) Luciferase reporters were co-transfected with miRNAs identical to miR-206, miR1, miR-140, as indicated. The 3'UTR-sensor constructs used are indicated below the columns. Relative luminescence units were plotted as a percentage of the control value obtained in absence of miRNA. A representative experiment of four independent experiments using two DNA plasmid preparations is shown. Error bars indicate s.d., $n=3$. Columns represent the wild-type or mutated $\left(\mathrm{TS}^{\mathrm{m}}\right) 3^{\prime} \mathrm{UTR}$ sensor co-transfected with miR-1 or miR-206 $(P<0.05, n=3)$, or with miR-140 (negative control). (B) Expression of endogenous proteins is regulated by miR-1 and/or miR-206 in NIH3T3 cells. Protein lysates were prepared from cells that were mock-transfected or transfected with miRNAs with or without antimiR-206, as indicated above each lane. Western blots were probed with the antibodies indicated next to each panel. (C) Northern blot showing the expression of mature miR-1 and miR-206 in differentiated $\mathrm{C} 2 \mathrm{C} 12$ cells. These miRNAs were not detected in NIH3T3 cells. U6 served as a loading control. (D) Northern blot showing inhibition of miR-1 and miR-206 expression in differentiated $\mathrm{C} 2 \mathrm{C} 12$ cells. Cells cultured in GM were transfected with antimiR-206 at concentrations indicated above each panel. Next, cells were cultured in DM for 5 days, RNA was isolated and probed against mature miR-1 and miR206, as well as U6; $50 \mathrm{nM}$ and $100 \mathrm{nM}$ antimiR-206 inhibited expression of both miR-1 and miR-206. is more similar to either D1-control or D1-miR-206 than to D2-control (Table 1). One possible interpretation of these results is that the repression of miR-206 partially restores the profiles observed at day 1 .

To assess the effect of the treatments on the expression level of putative miR-206 targets, we compared the differential expression empirical distributions of the set of genes containing putative target sites for miR-206 with that of all other genes (Fig. 1C,D). A putative target site was defined as a sequence complementary to the seed region of the miRNA (Rajewsky, 2006). We generated lists of target sites for perfect matches of six, seven and eight base pairs, required to mediate miRNA targeting (Grimson et al., 2007). Using the Mann-Whitney-Wilcoxon test we assessed the significance of the difference between the sets of genes containing each of these types of target sites and the set of genes without target site. The only significant results were obtained for the sets of genes containing 7mer and 8-mer matches, in the D2-antimiR and D2-control pairwise comparison ( $P$-values of 0.009 and 0.006 , respectively). The absence of significant differences between D1-control and D1miR-206 confirms the small effect of miR-206 overexpression on day one. The significant differences between D2-control and D2antimiR indicate that there is a large number of genes containing a putative target site that are de-repressed due to the inhibition of miR206 by antimiR-206 transfection.

We also examined the set of targets predicted by three major target prediction algorithms TargetScanS (TargetScan4.1)

Table 1. Spearman correlation coefficient between pairs of samples

\begin{tabular}{lcccc}
\hline & D1-control & D1-siRNA & D2-control & D2-antimiR \\
\hline D1-control & 1.00 & 0.96 & 0.91 & 0.95 \\
D1-siRNA & & 1.00 & 0.92 & 0.96 \\
D2-control & & & 1.00 & 0.92 \\
D2-antimiR & & & & 1.00 \\
\hline
\end{tabular}

(Friedman et al., 2009; Grimson et al., 2007; Lewis et al., 2005), PicTar (Krek et al., 2005) and miRanda (Griffiths-Jones et al., 2008) (supplementary material Fig. S1A). We repeated the comparison between the empirical distributions between the set of targets of each program and the set of genes not predicted to be targets (Fig. 1E,F). Once again, no significant difference was observed for the D1-miRNA and D1-control pairwise comparison. For the D2-antimiR and D2-control, the most significant difference was observed for the set of targets predicted by TargetScan $\left(P<10^{-8}, P<0.74\right.$ and $P<0.02$ for TargetScan, PicTar and miRanda, respectively).

To assess the impact of miR-206 on the expression profile not only through direct targeting but also through indirect effects we functionally annotated genes differentially expressed between D2-control and D2-antimiR. For genes upregulated in D2antimiR, that is, the set of genes that negatively correlates with the activity level of miR-206, and which therefore should include most direct targets, the list of significantly enriched annotation terms contained only generic terms, such as 'cell cycle' and 'acetylation'. For the set of genes downregulated in D2-antimiR (and that should constitute almost exclusively indirect targets) most of the enriched terms are related to muscle (supplementary material Table S1). This would suggest that miR-206 directly targets a relatively small number of genes that are repressors of a larger number of muscle-related genes (supplementary material Table S2 for the list containing these genes). This list includes for example myogenin, Laminin $\alpha 5$, Myh1, Myh3, Myh8, integrin $\alpha 7$, integrin $\beta 6$, fibrillin 1 , desmin, troponin 1 , troponins $\mathrm{T} 1$ and T3, dystroglycan 1, titin, actinin $\alpha 2$ amongst other interesting genes that are all associated with muscle structure and function.

\section{Experimental validation of targets for miR-206 and miR-1}

We generated a list of candidate miR-206 target genes that contained a seed site for miR-206 and were upregulated in the D2-antimiR sample or that were predicted to be miR-206 
targets by at least two of three target prediction databases (supplementary material Fig. S1). From these we selected putative targets associated with skeletal muscle development or differentiation. The transcripts of interest included Meox2, Fzd7 and RARB, which are known to be expressed in embryonic muscles, including developing limb muscles (Blentic et al., 2003; Cauthen et al., 2001; Mankoo et al., 2003; Mollard et al., 2000; Reijntjes et al., 2007), CLCN3, a chloride channel expressed in adult muscle and heart (Yamamoto-Mizuma et al., 2004), MAP4K3, a mitogen activated kinase primarily activating the JNK signalling pathway (Ramjaun et al., 2001) Smarcd2, Smarcb1, chromatin remodelling factors (Klochendler-Yeivin et al., 2006; Lickert et al., 2004; Wang et al., 1996), SH3BGRL3 (TIP-B1), a novel TNF inhibitory protein (Berleth et al., 2001) and NFAT5, a transcription factor expressed in myoblasts and regenerating muscles (Lopez-Rodríguez et al., 1999; O'Connor et al., 2007). The putative miR-206 and miR-1 target sites are conserved in human, mouse, rat and chicken, except for Smarcd2 where alignments showed that the seed sequence was poorly conserved (supplementary material Fig. S1). Sensor constructs were generated containing the 3'UTR with the potential microRNA binding site(s) (see Materials and Methods) and tested in luciferase assays. Chicken DF1 cells were transfected with 3'UTR-sensor constructs, Renilla luciferase vector and miR1 or miR-206, or with miR-140, which is not predicted to target these transcripts (Goljanek-Whysall et al., 2011) (Fig. 2A).

A known target, Connexin-43 (GJA1) (Anderson et al., 2006; Kim et al., 2006), served as a positive control and the GJA13'UTR sensor was regulated as predicted by miR-1 and miR-206. We defined validated targets as those that were efficiently downregulated by $\mathrm{miR}-1 / \mathrm{miR}-206$ but where the unrelated miR140 had no effect on the expression of the sensor construct. This approach confirmed Smarcd2, Smarcb1, MAP4K3, Fzd7, Meox2 and RARB as targets for both, miR-1 or miR-206. Interestingly, the 3 'UTRs of CLCN3 and SH3BGLR3 were regulated by miR206 but not miR-1. The NFAT5 sensor was only weakly regulated by miR-206 and miR-1. For all confirmed targets mutation of the putative target site in the $3^{\prime} \mathrm{UTR}$ rendered the sensor construct insensitive to miR-1 or miR-206 indicating that interaction with the target site is required (Fig. 2A).

Next, we investigated the ability of miR-1 and miR-206 to regulate the expression of target genes in a cellular context (Fig. 2B). We found that NIH3T3 cells expressed robust levels of most candidate targets (data not shown) but miR-1 and miR-206 were not expressed (Fig. 2C). Therefore these cells were ideal to investigate whether transfected miR-1 and miR-206 could regulate expression of the endogenous proteins. To determine if the effects of miR-1/miR-206 expression were specific we conducted rescue experiments with co-transfected antimiR-206, which suppressed the expression of both miR-1 and miR-206 in C2C12 myoblasts at $50 \mathrm{nM}$ and $100 \mathrm{nM}$ concentration (Fig. 2D).

Consistent with luciferase reporter assays CLCN3 protein level decreased after transfection with miR-206 but not after transfection with miR-1 compared to untransfected cells (Fig. 2B). The reduced levels of CLCN3 protein were restored by co-transfection of antimiR-206. Western blots of Smarcb1, Smarcd2, MAP4K3, Fzd7 and RARB showed decreased protein levels after transfection with miR-1 or miR-206 compared to untransfected cells (Fig. 2B). In all cases protein levels were restored to that of controls by co-transfection of antimiR-206. Meox 2 protein level was reduced by miR-1 but was not affected by miR-206 transfection, although in luciferase assays both miRs were effective in reducing expression levels. The decreased levels of Meox 2 protein could be restored by co-transfection of antimiR (Fig. 2B). Consistent with our results from luciferase assays NFAT5 protein levels were only slightly affected by transfection of miR-206 or miR-1 (Fig. 2B) and co-transfection of antimiR restored protein levels to that of controls.

\section{miRNA dependant downregulation of candidate genes during $\mathrm{C} 2 \mathrm{C} 12$ differentiation}

To determine whether interactions between miR-1/miR-206 with these novel targets are biologically relevant we examined their expression profiles during $\mathrm{C} 2 \mathrm{C} 12$ myoblast differentiation. When grown in differentiation medium (DM) containing low serum, $\mathrm{C} 2 \mathrm{C} 12$ cells differentiate into myotubes within 5 days and miR-1 and miR-206 are expressed by day 2 (Chen et al., 2006; Kim et al., 2006; Rao et al., 2006; Sweetman et al., 2008). Consistent with previous reports transfection of $\mathrm{C} 2 \mathrm{C} 12$ cells with miR-206 or miR-1 enhanced myogenic differentiation (Chen et al., 2006; Kim et al., 2006; Nakajima et al., 2006), whereas transfection of antimiRs inhibited both, miRNA expression (Fig. 2D) and myogenesis (data not shown).

Target gene expression was examined by QPCR and Western blots of differentiating $\mathrm{C} 2 \mathrm{C} 12$ cells in the presence or absence of antimiR-206 (Fig. 3A-C). Expression of Connexin-43 (GJA1) served as a positive control and, as previously reported by others, transcripts were downregulated significantly from day 2 of C2C12 cell myogenic differentiation (Kim et al., 2006); this was inhibited by blocking miRNA function through transfection of antimiR-206 (data not shown). Similarly, CLCN3, Smarcd2 and Smarcb1 transcripts were reduced during $\mathrm{C} 2 \mathrm{C} 12$ myogenic differentiation (Fig. 3A) and inhibition of miR-1/miR-206 with antimiR-206 prevented the downregulation of these transcripts and levels remained at $80-100 \%$ of that seen in undifferentiated cells at day 0 (Fig. 3B).

Transcripts for Meox2, RARB and NFAT5 were reduced during myogenesis from day 1 or day 2 onwards (Fig. 3A). However, downregulation of Meox2, RARB and NFAT5 transcript levels was only partially inhibited in the presence of antimiR-206 (Fig. 3B). This indicates that additional mechanisms, independent of $\mathrm{miR}-1 / \mathrm{miR}-206$ function, are involved in the negative regulation of Meox2, NFAT5 and RARB expression during $\mathrm{C} 2 \mathrm{C} 12$ cell differentiation.

There was no detectable change in Fzd7 and MAP4K3 transcript levels during $\mathrm{C} 2 \mathrm{C} 12$ cell myogenesis (Fig. 3A), indicating that miR-1/miR-206 may downregulate Fzd7 and MAP4K3 proteins mainly through inhibition of translation.

Next, we determined the effects of miR-1/miR-206 on protein expression in differentiating $\mathrm{C} 2 \mathrm{C} 12$ cells. Western blot analyses demonstrated that endogenous CLCN3, Smarcd2, MAP4K3, NFAT5 and RARB proteins were downregulated significantly during myogenic differentiation from day two onwards. Smarcb1, Fzd7 and Meox 2 protein levels were downregulated from day 3 or later (Fig. 3C). Transfection of antimiR-206, which blocks expression of miR-1/miR-206 in differentiating C2C12 cells (Fig. 2D; Fig. 3D), inhibited the downregulation of these target genes.

\section{Sustained expression of miR-1/miR-206 targets inhibits C2C12 cell myogenesis}

To investigate whether miRNA-mediated target repression is essential for myogenesis, we examined the consequences of 


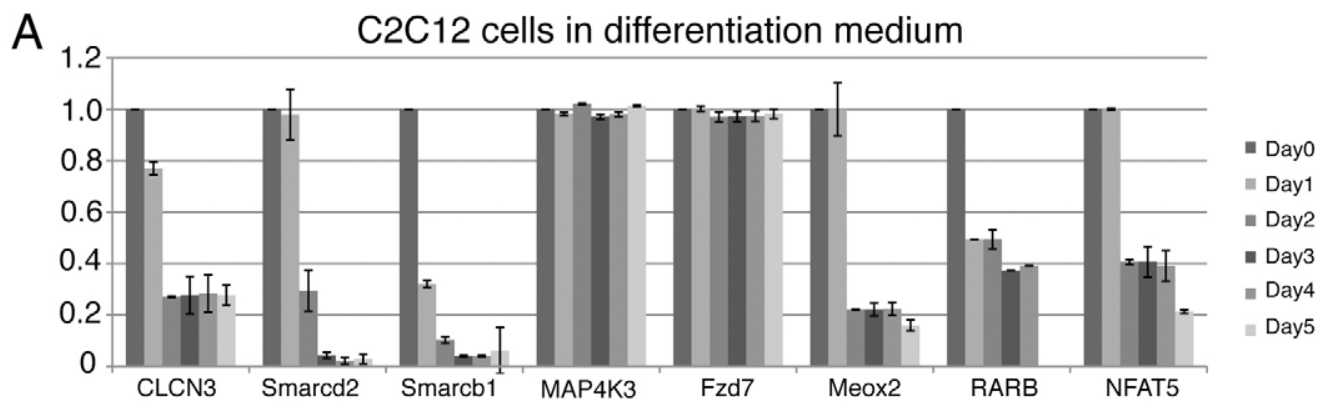

B C2C12 cells in differentiation medium plus antimir-206
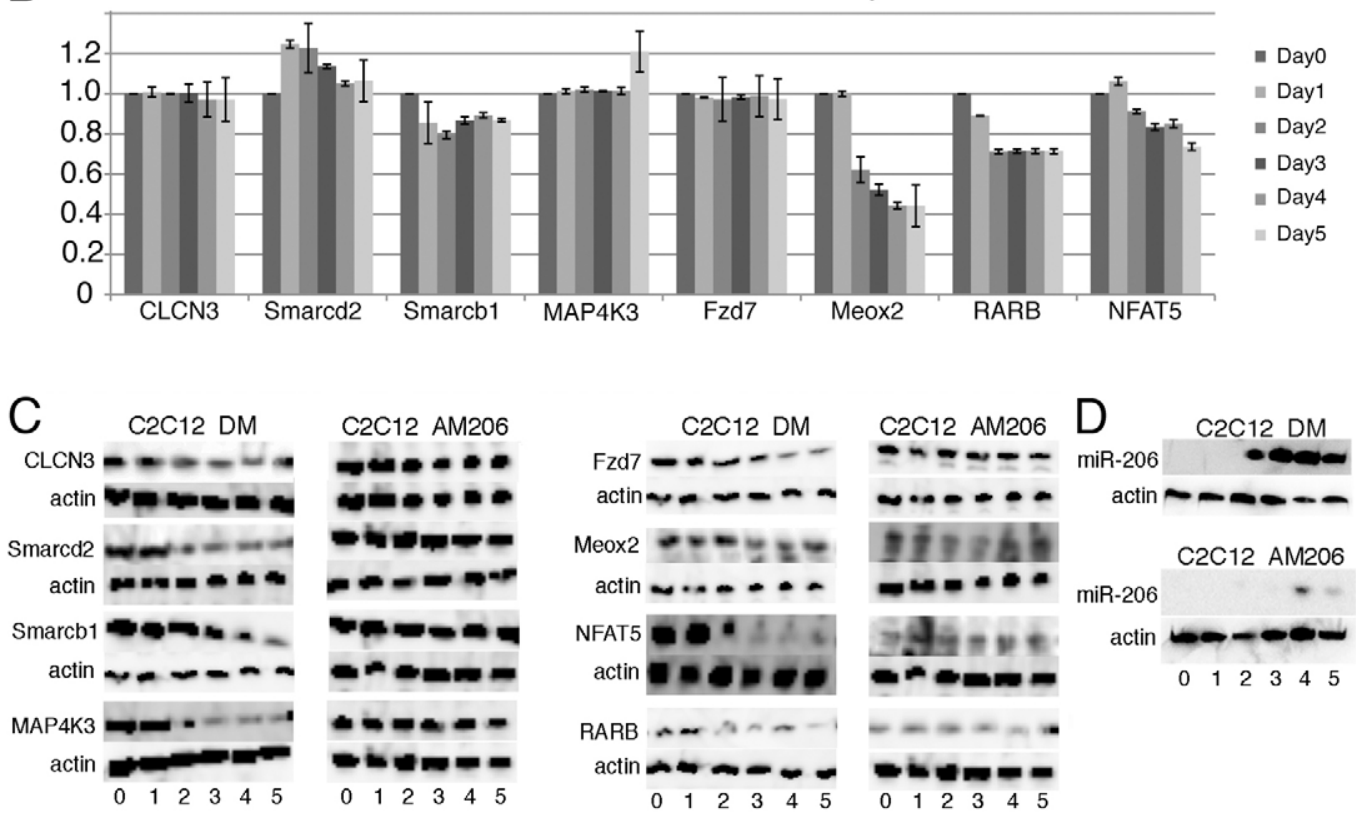

Fig. 3. Transcript and protein levels of candidate targets are regulated by miRs during C2C12 myogenesis. (A,B) qPCR for the transcripts indicated was performed on cDNA from $\mathrm{C} 2 \mathrm{C} 12$ myogenic cells on the days indicated by different shaded columns. Cells were grown in differentiation medium (DM) in the absence (A) or in the presence of antimiR (B), as indicated. Normalised values are plotted. (C) Protein lysate was harvested from C2C12 cells or from C2C12 cells treated with antimiR on the days indicated below each lane. Western blots were probed with the antibodies indicated next to each panel. Inhibition of miR-1/ miR-206 results in loss of downregulation of target genes. (D) Northern blot shows expression of miR-206 during C2C12 cell differentiation in the presence or absence of antimiR-206 as indicated. U6 served as loading control. AntimiR-206 was efficient in blocking miR-206 expression during differentiation.

forced expression of target genes. We transfected $\mathrm{C} 2 \mathrm{C} 12$ cells with eukaryotic expression constructs lacking the $3^{\prime} \mathrm{UTR}$. Based on GFP controls the transfection efficiency was around $30 \%$. Western blots showed that this led to significantly increased levels of target proteins. In all cases, elevated protein levels were still evident at day 5 of differentiation (supplementary material Fig. S2) and there was no evidence of increased apoptosis (supplementary material Fig. S4). After transfection $\mathrm{C} 2 \mathrm{C} 12$ were cultured in growth (GM) or differentiation medium (DM) and myogenic differentiation was assessed by immunostaining of myosin heavy chain (MyHC) with MF20 antibody (Fig. 4A,B). Mock-transfected $\mathrm{C} 2 \mathrm{C} 12$ cells cultured in GM did not differentiate until day 5 , when a few myotubes were detected due to depletion of growth factors. In contrast, after culture in DM, myotubes were observed at day 3 , and by day 5 most cells had differentiated and were labelled by immunostaining with MF20 (Fig. 4B). First we examined the effects of antimiR-206 transfection, which prevents effective downregulation of all target genes (Fig. 3C). This led to impaired myogenic differentiation, evidenced by fewer MF20 positive fibres (Fig. 4B) and by determining the fusion index and the total myotube area, which were both reduced. The cells transfected with antimiR-206 were more similar to cells grown in GM (Fig. 4C,D).

The antimiR-206 prevents downregulation of all miR-1/miR206 targets. Next we determined the effect of a single target on myogenic differentiation and expression plasmids were transfected individually. In most cases sustained target gene expression led to delayed myogenic differentiation of $\mathrm{C} 2 \mathrm{C} 12$ cells as shown by MF20 staining. Representative examples are shown (Fig. 4A). Following transfection with CLCN3, MAP4K3, Fzd7, SH3BGRL3, Smarcd2, Smarcb1 and NFAT5 only very few MF20 positive myotubes were detected after 3 days in DM (Fig. 4A and not shown) when compared to mock-transfected cells (Fig. 4B). After 5 days in DM myotubes were present in 


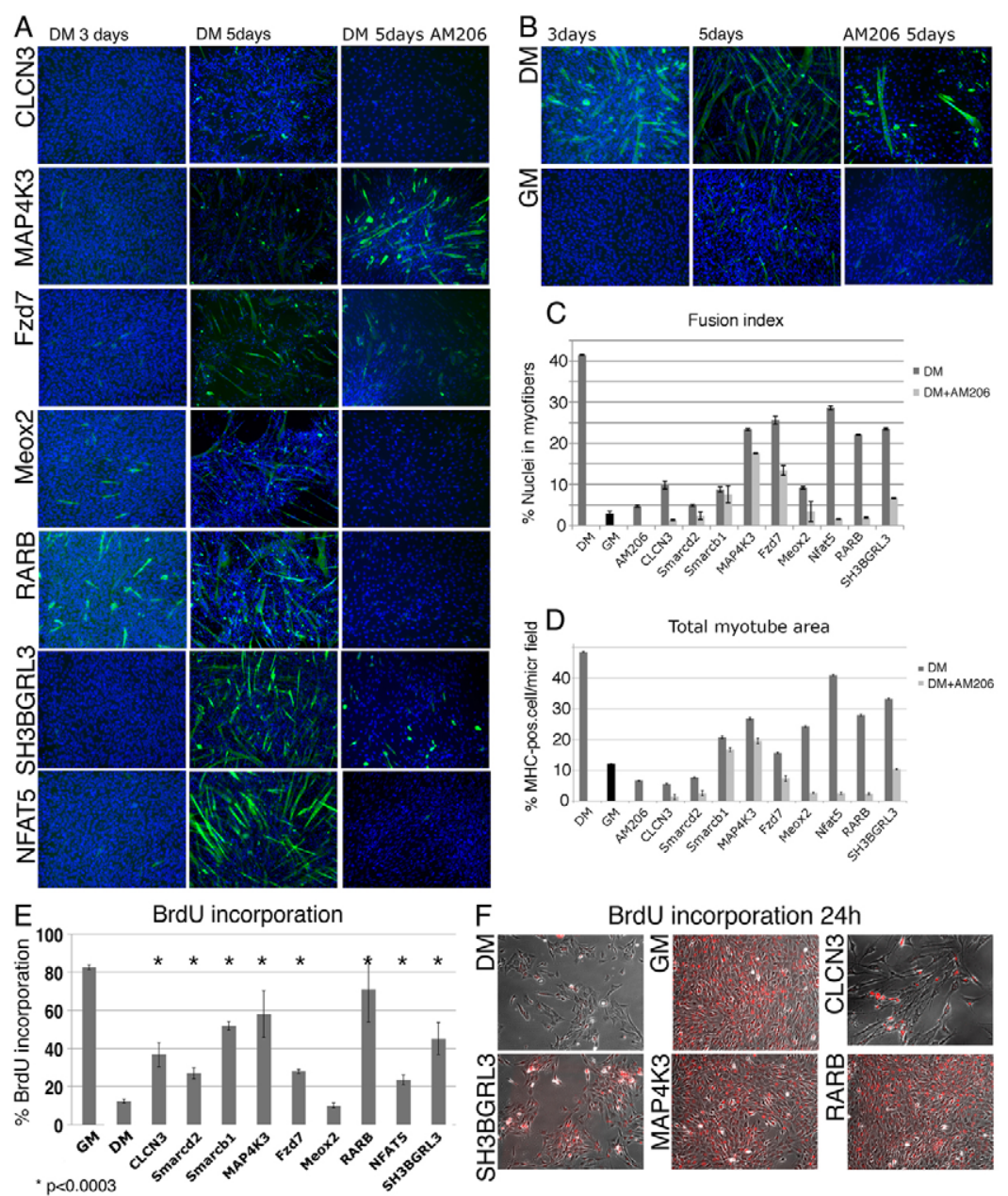

Fig. 4. Downregulation of target genes by miR-1 and miR206 is required for myogenic differentiation. $\mathrm{C} 2 \mathrm{C} 12$ cells were transfected with expression constructs encoding for target genes, as indicated. Following transfection, $\mathrm{C} 2 \mathrm{C} 12$ cells were cultured in differentiation medium (DM), as indicated.

(A) $\mathrm{C} 2 \mathrm{C} 12$ cells transfected with target genes or co-transfected with AM206 and target genes were cultured in DM and immunostained for MHC after 3 and 5 days of culture (MF20, green; DAPI, blue). (B) The staining obtained was compared with control mock-transfected cells cultured in DM, which express MHC (green) at day 3 and at day 5. By contrast, only few differentiated fibres (green) are present in cells cultured in GM for 5 days. Cells transfected with antimiR-206 (AM206) and cultured in DM show delayed myogenesis.

(C,D) Quantification of fusion index and total myotube area following transfection and culture of $\mathrm{C} 2 \mathrm{C} 12$ cells is shown. Overexpression of all target genes inhibited $\mathrm{C} 2 \mathrm{C} 12$ differentiation significantly $(P<0.05)$ with the exception of NFAT5. Cells overexpressing RARB and SH3BGRL3 differentiated efficiently compared with the DM control, probably due to their increased proliferation and consequent growth factor depletion from the culture medium. $\mathrm{C} 2 \mathrm{C} 12$ co-transfected with target genes and AM206 showed a greater delay in myogenesis. GM, growth medium; DM, differentiation medium. (E,F) Proliferation of $\mathrm{C} 2 \mathrm{C} 12$ cells following transfection with target genes and culture in DM was determined by anti-BrdU antibody (red). (E) The percentage of BrdU-positive cells is shown. Error bars indicate s.d. from three independent experiments. (F) $\mathrm{C} 2 \mathrm{C} 12$ overexpressing selected target genes and cultured in GM showed similar proliferation rate to $\mathrm{C} 2 \mathrm{C} 12$ cells cultured in $\mathrm{GM}$.

transfected $\mathrm{C} 2 \mathrm{C} 12$ cells, however their number was reduced, either strongly (MAP4K3, Fzd7, Meox2, RARB, Smarcb1) or weakly (SH3BGLR3, NFAT5), when compared to control cells (Fig. 4A,B). The negative effect on myogenic differentiation was confirmed by determining the fusion index and the total myotube area (Fig. 4C,D). The fusion index, determined by the number of nuclei per fiber divided by the number of total nuclei, was significantly reduced for most targets compared to control cells in DM (Fig. 4C). In addition, in most cases the percentage of total image area covered by myotubes (total myotube area) was significantly reduced $(P=0.05)$ with the exception of NFAT5 $(P=0.1)$. Co-transfection of antimiR-206 with the target genes enhanced the negative effect on myogenic differentiation (Fig. 4A,C,D), although the inhibition was sometimes not as great as that seen with antimiR-206 alone (see Discussion).

The removal of growth factors from the culture leads to cell cycle exit and activation of the $\mathrm{C} 2 \mathrm{C} 12$ myogenic differentiation program. To investigate whether miR-1/miR-206 targets affected C2C12 cell proliferation we measured BrdU incorporation (Fig. 4E,F). When cultured in GM $80 \%$ of cells incorporated BrdU compared to $12 \%$ of cells cultured in DM. Transfection of miR-1/miR-206 targets significantly increased the number of $\mathrm{C} 2 \mathrm{C} 12$ cells cultured in DM that incorporated BrdU, CLCN3 (38\%), Smarcd2 (28\%), Smarcb1 (52\%), MAP4K3 (58\%), Fzd7 (29\%), RARB (70\%), NFAT5 (25\%), SH3BGRL3 (46\%) (Fig. 4E). The exception was Meox2, which showed no significant increase in the number of BrdU-positive cells (Fig. 4E); representative images are shown (Fig. 4F). Together, these data show that downregulation of miR-1/miR-206 target genes in committed myoblasts is critical and failure to do so inhibits $\mathrm{C} 2 \mathrm{C} 12$ myogenic differentiation due to effects on different cellular processes such as proliferation and/or myoblast fusion.

We next asked whether inhibition of miR-1/miR-206 activity or overexpression of individual microRNA targets after the onset of $\mathrm{C} 2 \mathrm{C} 12$ differentiation could affect myogenesis. $\mathrm{C} 2 \mathrm{C} 12$ cells were cultured in DM and transfected on day 3 with either antimiR-206 or with target gene expression constructs (transfection efficiency was 10-15\%). After a further 3 days of culture in DM myogenic differentiation was analysed by staining with MF20 antibody and determination of fusion and differentiation indices (Fig. 5A-C). C2C12 cells transfected with antimiR-206, which inhibits miR-1/miR-206 activity and affects all targets, resembled cells cultured in GM and fewer MF20 positive myotubes were seen compared to control cells cultured in DM (Fig. 5A). The fusion index and total myotube area were both reduced and were similar to cells cultured in GM (Fig. 5B,C). Next we determined the effect of individual target genes. Transfection of expression constructs on day 3 led to impaired myogenesis to varying degrees by day 6 . The most dramatic effects were observed with CLCN3 and Meox2; however, expression of all other targets after the onset of 
A

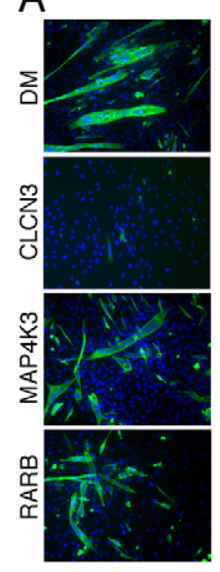

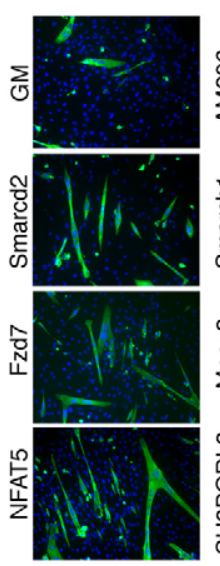

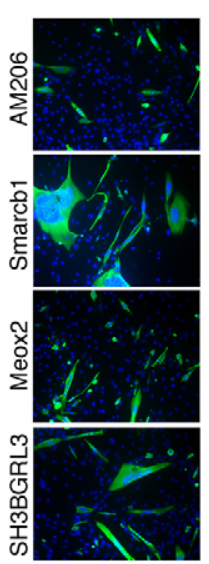

B
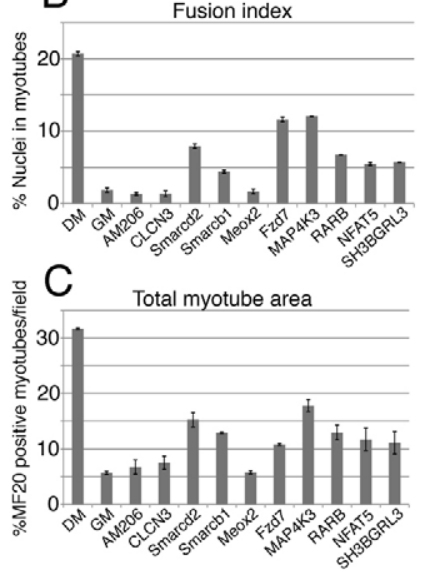

Fig. 5. Downregulation of target genes by miR-1 and miR206 is required for terminal myogenic differentiation. $\mathrm{C} 2 \mathrm{C} 12$ cells were transfected with expression constructs encoding for the indicated target genes at day 3 of differentiation when myotubes started to form. (A) Following transfection, $\mathrm{C} 2 \mathrm{C} 12$ cells were cultured in differentiation medium (DM) until day 6 and then immunostained for MHC (MF20, green; DAPI, blue). (B,C) Quantification of fusion index and total myotube area is shown. Overexpression of all target genes inhibited terminal differentiation of committed C2C12 cells significantly $(P<0.05)$. Cells transfected with Smarcb1 also displayed unusual morphology.
$\mathrm{C} 2 \mathrm{C} 12$ cell myogenesis led to reduced fusion and total myotube area.

\section{C2C12 myoblasts activate alternative differentiation programs after sustained expression of miR-1/miR-206 target genes}

$\mathrm{C} 2 \mathrm{C} 12$ and primary myoblasts can transdifferentiate into osteogenic or adipogenic cells following treatment with bone morphogenic proteins (BMPs) or adipogenic inducers, respectively (Asakura et al., 2001; Nakajima et al., 2006). To investigate whether miR-1/miR-206 targets can promote alternative differentiation programs, we used quantitative RTPCR and histological stains to examine osteogenesis, adipogenesis and chondrogenesis (Fig. 6). Osteogenic markers included early and late markers, osterix and osteocalcin, as well as alkaline phosphatase staining. Collagen 10A (Col10A) and the matrix metalloproteinase, MMP13 as well as Alcian Blue staining were used to indicate chondrogenic differentiation. TM4SF1 and leptin were used to assay for adipogenesis.

None of the microRNA-regulated genes reported here were able to induce adipogenesis-related gene expression in $\mathrm{C} 2 \mathrm{C} 12$ cells (data not shown). In contrast, osterix and osteocalcin were detected
A

Osteogenesis

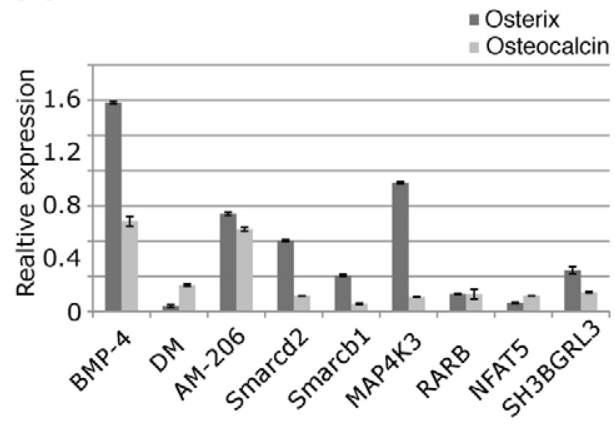

B

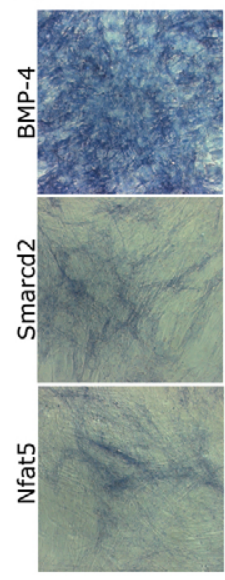

Alkaline Phosphatase

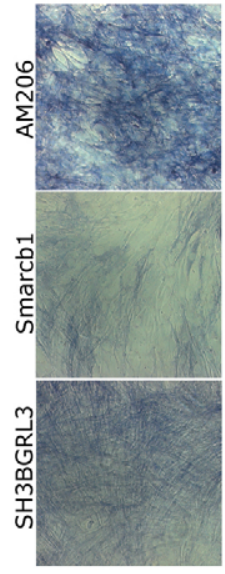

C Chondrogenesis Col10A $=\mathrm{MMP} 13$

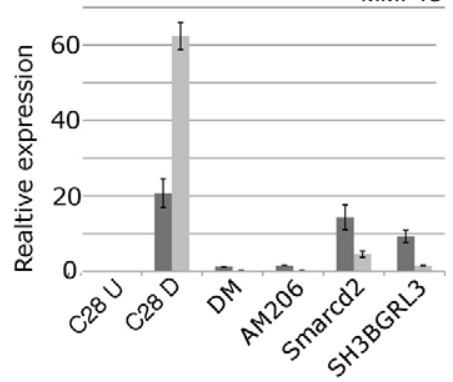

D Alcian blue

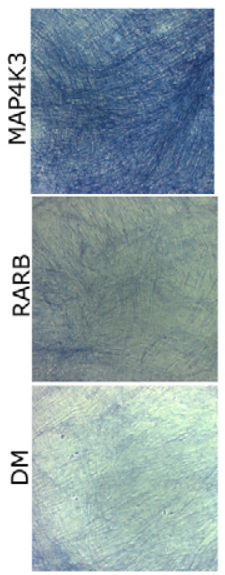

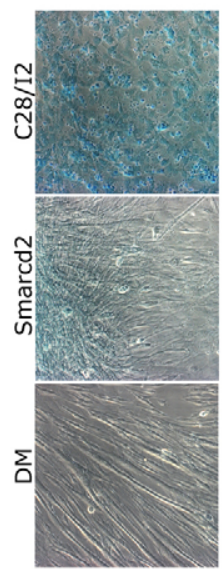

Fig. 6. Overexpression of target genes in $\mathrm{C} 2 \mathrm{C} 12$ cells leads to expression of chondrogenic and/or osteogenic markers. $\mathrm{C} 2 \mathrm{C} 12$ cells were transfected with target genes and cultured in DM. (A,C) q-PCR showed that expression of AM206 or selected target genes led to increased expression of osteogenic (A) and chondrogenic (C) markers, compared with cells cultured in DM. (A) Expression of AM206 and all target genes shown led to significantly increased expression $(P<0.05)$ of the early osteogenic marker osterix compared with mock-transfected control cells and some genes also increased expression of the late osteogenic marker, osteocalcin. (B) $\mathrm{C} 2 \mathrm{C} 12$ cells transfected with AM206 or the target genes indicated showed positive alkaline phosphatase staining compared with controls. $\mathrm{C} 2 \mathrm{C} 12$ cells treated with BMP-4 served as a positive control for osteogenic differentiation. Smarcd2, Smarcb1, RARB and NFAT5 were less efficient in inducing osteogenesis compared with AM206- or MAP4K3transfected cells and only $\sim 10 \%$ of cells stained positive for alkaline phosphatase. (C) Relative expression levels of chondrogenic markers: Col10A and MMP13 in cells transfected with Smarcd2 or SH3BGRL3 were significantly higher $(P<0.05)$ than in control $\mathrm{C} 2 \mathrm{C} 12$ cells or undifferentiated C28/I2 cells. Transfection of AM206 did not result in increase of chondrogenic markers. Differentiated C28/I2 cells served as a positive control. (D) Alcian Blue staining indicating chondrogenic differentiation. Approximately $20 \%$ of cells displayed positive Alcian Blue staining after transfection with Smarcd2 or SH3BGRL3. 
in $\mathrm{C} 2 \mathrm{C} 12$ cells cultured in DM and transfected with antimiR-206 or expression plasmids encoding Smarcd2, Smarcb1, MAP4K3 or SH3BGRL3 (Fig. 6A). Transfection of RARB and NFAT5 plasmids also led to expression of osteogenic markers, however, at relatively low levels. Histological staining for alkaline phosphatase resulted in a strong signal, comparable to that in control cells cultured in BMP-4, or in cells transfected with antimiR-206 or MAP4K3 (Fig. 6B). The chondrogenic markers MMP13 and Cola10A were significantly increased in $\mathrm{C} 2 \mathrm{C} 12$ cells transfected with Smarcd2 or SH3BGRL3 (Fig. 6C) and these cells also stained positive for Alcian Blue (Fig. 6D). We used differentiated chondrocytes, C28/I2, as control.

Finally, we investigated whether transfection of $\mathrm{C} 2 \mathrm{C} 12$ cells on day 3 of differentiation could activate osteogenic or chondrogenic gene expression after a further three days culture (supplementary material Fig. S3). Surprisingly, some alkaline phosphatase positive cells were detected after transfection of antagomiR-206 or transfection of expression plasmids encoding Smarcd2 or SH3BGRL3. Alcian Blue signal was seen in very small patches of $\mathrm{C} 2 \mathrm{C} 12$ cells after transfection with SH3BGRL3. These data indicate that inappropriate expression of at least some miR-1/miR-206 target genes can promote cell fates closely related to but distinct from skeletal muscle.

\section{Discussion}

Skeletal myogenesis serves as an important paradigm for cell differentiation and the muscle differentiation program is recapitulated in $\mathrm{C} 2 \mathrm{C} 12$ cells. To determine the role of miR-1/ miR-206 during skeletal myogenesis, we identified potential targets based on mRNA profiling following manipulation of miR206 activity in $\mathrm{C} 2 \mathrm{C} 12$ cells, predictions by multiple algorithms and prior knowledge of genes known to be involved in myogenesis. This approach has helped to avoid false positive hits; however, it is likely that there are additional targets that have yet to be confirmed. We focused on targets that were more likely to be biologically relevant and most of the targets selected for further study have been implicated in skeletal or cardiac myogenesis. Using a number of complementary assays we validated nine novel miR-1/miR-206 targets from our screen.

Interestingly, inhibiting miRNA function by antimiR-206 affected the expression of a greater number of genes in $\mathrm{C} 2 \mathrm{C} 12$ cells than overexpression of miR-206 (Fig. 1A,B). This may indicate that many miR-206 targets are not yet expressed on day one of differentiation and therefore increasing the level of miR206 had a limited effect. Conversely, some target genes involved in embryonic myogenesis may no longer be expressed in $\mathrm{C} 2 \mathrm{C} 12$ myoblasts, which are derived from adult satellite cells. An example of this is Pax3, which we showed recently, is targeted by miR-206 during the progenitor to myoblast transition (GoljanekWhysall et al., 2011) but was not identified in this new screen, although it has been reported that Pax 3 and the highly related Pax 7 are regulated by miR-1/miR-206 during satellite cell activation (Chen et al., 2010; Hirai et al., 2010).

The finding that the expression profile obtained from the D2antimiR-206 sample was similar to D1 control suggests that repression of miR-206 partially restores day 1 expression profiles (Table 1). Functional annotation of genes differentially expressed between D2-control and D2-antimiR-206 showed that genes downregulated in the D2-antimiR-206 group, so-called antitargets, were enriched for terms related to muscle (supplementary material Table S1). This suggests that miR-206 directly targets, and negatively regulates a relatively small number of genes, which would otherwise repress a larger number of muscle-related genes (supplementary material Table S2 for the list containing these genes). This is consistent with previous findings, which reported that after introduction of miR-1/miR-206 a significant number of upregulated genes were related to muscle structure and function. Similar to our observations the authors suggested that this is most likely due to indirect effects after the primary differentiation-inducing stimuli of the miRNAs (Kim et al., 2006).

We validated candidate genes using in vitro and cell based approaches. miR-1 and miR-206 downregulated luciferase sensor constructs whilst introduction of specific mutations into the potential miR target site showed that this was dependant on the presence of these conserved target sites in the $3^{\prime}$ UTR of the respective genes (Fig. 2A). Notably not all confirmed target sites are equally regulated by miR-1 and miR-206. For example, the target site we identified in CLCN3 is regulated by miR-206 but not miR-1 while the Meox 2 site is more strongly affected by miR-1 than miR-206. Furthermore, transfection of miR-206 and miR-1 led to a significant reduction of the putative target proteins in NIH3T3 cells, whilst co-transfection with antimiRs restored protein expression to normal levels (Fig. 2B). This indicates that $\mathrm{miR}-1 / \mathrm{miR}-206$ can interact with endogenous mRNAs in a cellular context.

During $\mathrm{C} 2 \mathrm{C} 12$ cell myogenic differentiation miR-206 and miR-1 are highly expressed from day 2 onwards (Sweetman et al., 2008; Chen et al., 2006; Kim et al., 2006; Rao et al., 2006) and, consistent with previous observations, inhibition of $\mathrm{miR}-1 / \mathrm{miR}$ 206 activity led to impaired myogenesis (Fig. 4B) (Chen et al., 2006). Increased microRNA expression was associated with reduced expression of the novel targets in $\mathrm{C} 2 \mathrm{C} 12$ cells (Fig. 3A,C) and suggests that downregulation of these genes may be required to facilitate myogenic differentiation. This was confirmed by sustained expression of target genes in $\mathrm{C} 2 \mathrm{C} 12$ cells undergoing differentiation following plasmid transfection. All expression plasmids led to a significant increase of protein levels compared to normal endogenous levels (supplementary material Fig. S2) and these increased protein levels were still seen at day 5 of differentiation. Transfection of validated miR-1/miR-206 targets had a negative effect on myogenesis, which was further enhanced by co-transfection of antimiR-206 in all cases. However, co-transfection of both target gene expression plasmid plus antimiR-206 was sometimes less effective than transfection with antimiR-206 alone. There are different possible explanations for this. The transfection of plasmid DNA plus antimiR may lead to less efficient uptake of antimiR. We also show that sustained overexpression of target genes increased proliferation (Fig. 4E,F). Therefore cells reached confluency faster and cultures were depleted of growth factors, which can then promote spontaneous differentiation. This would ameliorate the negative effects on myogenesis.

The inhibitory effects varied from almost complete inhibition of myotube formation (e.g. CLCN3) to only slight phenotypic changes, such as formation of thinner fibres containing a smaller number of nuclei (e.g. Fzd7, RARB) (Fig. 4A,B). This was quantified by determination of fusion index and total myotube area (Fig. 4C,D) using established approaches (Ricotti et al., 2011). To examine possible effects of miR-1/miR-206 inhibition after the onset of differentiation, we modified miRNA levels, by antimiR-206 transfection at day 3 (in DM). At this point $\mathrm{C} 2 \mathrm{C} 12$ 
myoblasts have elongated and begun to fuse and express sarcomeric myosin, detectable with MF20 immunostaining. They also express high levels of miR-1/miR-206 (e.g. Chen et al., 2006; Sweetman et al., 2008) (Fig. 3D; Fig. 4B). However, after antimiR-206 transfection both fusion index and total myotube area were dramatically reduced and were similar to that of cells kept in growth medium (Fig. 5B,C). Despite low transfection efficiency, transfection of individual target genes at day 3 also negatively affected $\mathrm{C} 2 \mathrm{C} 12$ cell myogenesis, with the strongest effects apparent for the CLCN3 chloride channel (Fig. 5A). For RARB, NFAT5 and SH3BGRL3 the negative effect on myogenesis appeared to be stronger when cells were transfected at day 3 compared to transfection at day 0 . At present we cannot explain the reasons for this observation. However, it is possible that this is related to specific functions of these genes, which are currently not well understood.

Together our results showed that modifying miRNA activity or the levels of single targets had a negative effect on myogenesis, even when performed after the onset of differentiation. This illustrates that miRNA function is pivotal for the successful execution and maintenance of the differentiation program. In addition, most of the genes investigated enhanced cell proliferation when introduced into C2C12 cells cultured in differentiation medium (Fig. 4E,F).

To establish whether any of the validated miR-1/miR-206 targets are associated with alternative differentiation programs we examined the expression of adipogenic, chondrogenic and osteogenic markers. miR-206 has been shown to be expressed in osteoblasts; however, it needs to be downregulated to facilitate their differentiation (Inose et al., 2009). This is consistent with data from $\mathrm{C} 2 \mathrm{C} 12$ cells, which no longer express miR-1 after induction of osteoblastic differentiation (Nakajima et al., 2006). Whist the importance of miR-1/miR-206 for myogenic differentiation and maturation is widely recognised, it has been suggested that miR-1/ miR-206 are less important for cell fate determination (Chen et al., 2006; Nakajima et al., 2006). Interestingly, we show here that inhibition of miR-1/miR-206 activity or sustained expression of a number of novel target genes not only interferes with myogenesis (Figs 4,5) but can also lead to the activation of osteogenic and chondrogenic markers (Fig. 6, supplementary material Fig. S3). These findings confirm that miR-1 and miR-206 activity is integral to myogenesis at both the early commitment stages (GoljanekWhysall et al., 2011) and during the subsequent differentiation process. We propose a dual function whereby miRNA mediated downregulation of genes, which would enhance non-myogenic programs and/or promote proliferation, is crucial for both the complete cellular commitment as well as efficient execution of the differentiation program. This is similar to our recent observations in developing embryos, where we showed that miR-206 contributes to the complete silencing of Pax-3, a pro-myogenic gene expressed in skeletal muscle progenitor cells (GoljanekWhysall et al., 2011). This downregulation facilitates the transition of progenitor to committed myoblast, and similar regulatory interactions are also important during the activation of satellite cells (Chen et al., 2010; Hirai et al., 2010).

Thus, miR-1 and miR-206 represent a core mechanism ensuring efficient cell fate commitment and myogenic differentiation, in developing embryos, satellite cells and in $\mathrm{C} 2 \mathrm{C} 12$ myoblasts. Our study identifies a series of novel targets for miR-1/miR-206, and demonstrates that their coordinated downregulation is required to prevent continued myoblast proliferation and the expression of genes associated with alternative cell fates.

\section{Materials and Methods}

\section{DNA constructs and luciferase assay}

The modified pGL3 vector (Promega) (Tuddenham et al., 2006) contained 3' UTR regions that were PCR amplified from chick embryo cDNA (HH stage 17-26). To mutate the predicted target sites a BamHI site (miR-1/miR-206 target sites) was introduced, which led to point mutations and facilitated identification of clones. A list of primers used is available in supplementary material Table S3.

DF1 chicken dermal fibroblasts were cultured in 96-well plates and transfected using Lipofectamine 2000 (Invitrogen) with either WT or mutant sensor constructs (200 ng), with or without miR-206/miR-1 (50 nM) (Sigma) as described (Goljanek-Whysall et al., 2011). The empty pGL3 vector or transfection with miR-140, which is not predicted to target any of the genes screened were used as a negative controls. Each experiment had triplicate samples and was carried out four times using two independent plasmid preparations of each reporter. Protein was extracted after 48 hours, luciferase activity was measured using a multilabel counter (Victor2, Perkin-Elmer, MA) and normalised to Renilla luciferase. Relative reporter activity for miR treated cells was obtained by normalisation to non-miR-treated WT or mutant constructs, respectively. $P$-values were calculated by Student's $t$-test.

\section{Cell culture, western blots and analysis of cell differentiation}

Mouse NIH3T3 cells were cultured in DMEM supplemented with $10 \%$ FBS and $1 \%$ penicillin/streptomycin. NIH3T3 cells were transfected with miR-206 or miR1 (50 nM, Sigma), with or without antimiR-206 (100 nM, Ambion) using Lipofectamine 2000. Protein was extracted after 48 hours following standard protocols using lysis buffer with protease and protein phosphatase inhibitors (Roche), $20 \mu \mathrm{g}$ cell lysate was run on $4-12 \%$ polyacrylamide gels and blotted onto nitrocellulose using a semidry blotter (Bio-Rad). Primary antibodies (1:500 or 1:1000) and secondary antibodies coupled to HRP (Jackson Laboratories) were applied for 1 hour each at room temperature. Primary antibodies used: CLCN3 (LifeSpan Biosciences), Meox2, SMARCD2, SMARCB1, NFAT5, RARB, caspase-9 and actin (Abcam, Cambridge, UK).

Mouse $\mathrm{C} 2 \mathrm{C} 12$ cells were maintained in growth medium (GM: DMEM, 10\% FBS, 1\% penicillin-streptomycin). Myogenic differentiation was induced in subconfluent cells in differentiation medium (DM: DMEM, 2\% horse serum, 1\% penicillin-streptomycin) as described (Sweetman et al., 2008). After transfection with antimiRs (50 nM, Ambion), cells were incubated in DM. Whole cell lysates were extracted on the day of transfection and then every 24 hours for 5 days. Osteogenic differentiation of $\mathrm{C} 2 \mathrm{C} 12$ cells was induced in DMEM supplemented with $5 \%$ horse serum and $400 \mathrm{ng} / \mathrm{ml}$ human recombinant BMP-4 for 6 days as described (Katagiri et al., 1994). To examine alkaline phosphatase activity histochemically, cells were fixed and stained as described (Katagiri et al., 1994).

C28/I2 cells were cultured and induced to chondrogenic differentiation as described previously (Greco et al., 2011). Alcian Blue staining was performed as previously described (Swingler et al., 2012) and C28/I2 cells served as positive control. For adipogenic differentiation, $\mathrm{C} 2 \mathrm{C} 12$ cells were grown to confluency in GM and switched to an adipogenic medium as described (Akimoto et al., 2005). Apoptosis was induced by $2 \mathrm{mM} \mathrm{H}_{2} \mathrm{O}_{2}$ for 6 hours as described (Salucci et al., 2010).

To determine the $\mathrm{C} 2 \mathrm{C} 12$ fusion index we counted the number of nuclei in myotubes and divided this by the total number of nuclei, after multiplication by 100 the $y$-axis in Fig. 4C, Fig. 5B represents percent of nuclei within myotubes. The total myotube area represents the percent of total image area covered by myotubes. Both were established using six different microscopic fields from three different experiments using ImageJ.

\section{Microarray samples and annotation methods}

We chose two time points that showed the highest differences in mRNA levels of a known miR-206 target (GJA1). Control C2C12 cells were cultured in DM and harvested at day 1 or day 2 (D1 control, D2 control). To activate miR-206 prematurely, cells were transfected with miR-206 and RNA was harvested at day 1 (D1 miRNA group). To inhibit miR-206, C2C12 cells were treated with antimiR206 and harvested at day 2 of differentiation (D2 antimiR-206 group). Three replicate samples of RNA were harvested and Illumina's MouseWG-6 V2 BeadChip microarrays were probed. Pre-processing of microarray data was performed using $\mathrm{R}$ and Bioconductor. Normalisation was performed using the lumi package (Du et al., 2008). Microarray probes were annotated using the lumiMouseIdMapping package. 3'UTR sequences were downloaded from Ensembl. Functional annotation was performed using the DAVID webserver (Huang et al., 2008). The data was submitted to GEO (accession number GSE34069) and meets the MIAME requirements.

\section{RNA isolation and northern analysis}

Standard methods were used as described previously (Sweetman et al., 2008).

\section{Quantitative real time PCR}

cDNA was generated using random hexamers and Superscript II reverse transcriptase (Invitrogen). TaqMan primers for real-time PCR reactions were 
obtained from Applied Biosystems as follows: Meox2: Mm00801881_m1; NFAT5 Mm00467257_m1; RARb: Mm01319674_m1; GJA1: Mm00439 105 m1; CLCN3: Mn01348786_m1; Evi1: Mm00514810_m1; SMARCB1: Mm00448 776_m1 SMARCD2: Mm00473467_g1. 18S ribosomal RNA was used as an internal control to normalise for differences in the amount of total RNA in each sample. TaqMan $18 \mathrm{~S}$ primers and a $5^{\prime}$-VIC-labelled probe were used according to the manufacturer's instructions (Applied Biosystems). Each experiment had triplicate samples and the values for day $1-5$ were normalised to the value obtained for day 0 Cell fate was analysed using SYBR Green qPCR SuperMix (Invitrogen) and following primers: OsterixF: 5'-GGGTTAAGGGGAGCAAAGTCAGAT-3', OsterixR: 5'-CTGGGGAAAGGAGGCACAAAGAAG-3', OsteocalcinF: 5'-CTGAGTCTGACAAAGCCTTC-3', OsteocalcinR: 5'-GCTGTGACATCCATACTTGC-3', Col10F: 5'-ATTATCCACTGCTCCTGGGCAGA-3', Col10R: 5'-ATAGATTCTGGTGCTGGGAGATGCG-3', MMP13F: 5'-AAAGACAGATTCTTCTGGCGCCTG- $3^{\prime}$. Mouse $\beta$-actin was used as reference gene.

\section{Expression vectors for validated target genes and BrdU incorporation assay} Expression plasmids encoding target genes were obtained from Source Bioscience Life Sciences (I.M.A.G.E. full length cDNAs). The expression vectors were pCMV.Sport 6 (4396bp) and pCMV.Sport6.1 (4177bp), empty pCMV.Sport6 served as control. The I.M.A.G.E. clone numbers are as follows, Smarcd2 2647494, Smarcb1: 3154501, CLCN3: 6833732, Fzd7: 6844727, Meox2: 008594 MAP4K3: 6825201, RARB: 30608242, NFAT5: 40083218, SH3BGRL3: 341987. $\mathrm{C} 2 \mathrm{C} 12$ cell proliferation was assessed by BrdU incorporation as described (Shan et al., 2007). Briefly, C2C12 cells were seeded on glass coverslips and transfected as described (Sweetman et al., 2008). Control cells were treated with lipofectamine only. After $4 \mathrm{~h}$ incubation cells were labelled with $10 \mu \mathrm{M}$ of BrdU (SigmaAldrich) for $3 \mathrm{~h}$ and harvested after an additional $24 \mathrm{~h}$. Cells were fixed in 4\% paraformaldehyde and stained with BrdU antibody $(1: 100)$ and donkey anti-mouse IgG (FITC conjugated, 1:250; Jackson ImmunoResearch Laboratories). BrdU quantification was assessed by analysing 6 different microscopic fields from three different experiments by dividing the number of nuclei stained positive with antiBrdU antibody by the total number of nuclei. $\mathrm{C} 2 \mathrm{C} 12$ cell differentiation was assessed by MF20 staining (Sweetman et al., 2008). Images were analysed using Axiovision and ImageJ. $P$-values were calculated by Student's $t$-test.

\section{Acknowledgements}

We thank Nick Redshaw for help with luciferase assays and molecular cloning, Caroline Pennington for help with qPCR (Taqman), Tracey Swingler and Orla Jupp for providing C28/I2 cells and protocols, and Vince Moulton and Grant Wheeler for discussions.

\section{Funding}

K.G., H.P. and D.S. were funded by the Biotechnology and Biological Sciences Research Council [grant numbers BB/D016444, BB H019979] to A.M. T.R. was funded by an EU FP6 network [SCIROCCO, LSHG-CT-2006-037900] of which T.D. was a member

Supplementary material available online at

http://jcs.biologists.org/lookup/suppl/doi:10.1242/jcs.101758/-/DC1

\section{References}

Akimoto, T., Ushida, T., Miyaki, S., Akaogi, H., Tsuchiya, K., Yan, Z., Williams, R. S. and Tateishi, T. (2005). Mechanical stretch inhibits myoblast-to-adipocyte differentiation through Wnt signaling. Biochem. Biophys. Res. Commun. 329, 381385 .

Anderson, C., Catoe, H. and Werner, R. (2006). MIR-206 regulates connexin43 expression during skeletal muscle development. Nucleic Acids Res. 34, 5863-5871.

Asakura, A., Komaki, M. and Rudnicki, M. (2001). Muscle satellite cells are multipotential stem cells that exhibit myogenic, osteogenic, and adipogenic differentiation. Differentiation 68, 245-253.

Bartel, D. P. (2004). MicroRNAs: genomics, biogenesis, mechanism, and function. Cell 116, 281-297.

Bartel, D. P. (2009). MicroRNAs: target recognition and regulatory functions. Cell 136, 215-233.

Berleth, E. S., Masso-Welch, P. A., Kazim, L. A., Ip, M. M., Mihich, E. and Ehrke, M. J. (2001). Expression, tissue distribution, and cellular localization of the antiapoptotic TIP-B1 protein. J. Leukoc. Biol. 69, 995-1005.

Blentic, A., Gale, E. and Maden, M. (2003). Retinoic acid signalling centres in the avian embryo identified by sites of expression of synthesising and catabolising enzymes. Dev. Dyn. 227, 114-127.

Carthew, R. W. (2006). Gene regulation by microRNAs. Curr. Opin. Genet. Dev. 16, 203-208.

Cauthen, C. A., Berdougo, E., Sandler, J. and Burrus, L. W. (2001). Comparative analysis of the expression patterns of Wnts and Frizzleds during early myogenesis in chick embryos. Mech. Dev. 104, 133-138.
Chen, J. F., Mandel, E. M., Thomson, J. M., Wu, Q., Callis, T. E., Hammond, S. M., Conlon, F. L. and Wang, D. Z. (2006). The role of microRNA-1 and microRNA-133 in skeletal muscle proliferation and differentiation. Nat. Genet. 38, 228-233.

Chen, J. F., Tao, Y., Li, J., Deng, Z., Yan, Z., Xiao, X. and Wang, D. Z. (2010) microRNA-1 and microRNA-206 regulate skeletal muscle satellite cell proliferation and differentiation by repressing Pax7. J. Cell Biol. 190, 867-879.

Darnell, D. K., Kaur, S., Stanislaw, S., Konieczka, J. H., Yatskievych, T. A. and Antin, P. B. (2006). MicroRNA expression during chick embryo development. Dev. Dyn. 235, 3156-3165.

Du, P., Kibbe, W. A. and Lin, S. M. (2008). lumi: a pipeline for processing Illumina microarray. Bioinformatics 24, 1547-1548.

Friedman, R. C., Farh, K. K., Burge, C. B. and Bartel, D. P. (2009). Most mammalian mRNAs are conserved targets of microRNAs. Genome Res. 19, 92-105.

Goljanek-Whysall, K., Sweetman, D., Abu-Elmagd, M., Chapnik, E., Dalmay, T., Hornstein, E. and Münsterberg, A. (2011). MicroRNA regulation of the paired-box transcription factor Pax 3 confers robustness to developmental timing of myogenesis. Proc. Natl. Acad. Sci. USA 108, 11936-11941.

Greco, K. V., Iqbal, A. J., Rattazzi, L., Nalesso, G., Moradi-Bidhendi, N., Moore, A. R., Goldring, M. B., Dell'Accio, F. and Perretti, M. (2011). High density micromass cultures of a human chondrocyte cell line: a reliable assay system to revea the modulatory functions of pharmacological agents. Biochem. Pharmacol. 82 , 1919-1929.

Griffiths-Jones, S., Saini, H. K., van Dongen, S. and Enright, A. J. (2008). miRBase: tools for microRNA genomics. Nucleic Acids Res. 36, D154-D158.

Grimson, A., Farh, K. K., Johnston, W. K., Garrett-Engele, P., Lim, L. P. and Bartel, D. P. (2007). MicroRNA targeting specificity in mammals: determinants beyond seed pairing. Mol. Cell 27, 91-105.

Guo, H., Ingolia, N. T., Weissman, J. S. and Bartel, D. P. (2010). Mammalian microRNAs predominantly act to decrease target mRNA levels. Nature 466, 835-840

Hinits, Y., Williams, V. C., Sweetman, D., Donn, T. M., Ma, T. P., Moens, C. B. and Hughes, S. M. (2011). Defective cranial skeletal development, larval lethality and haploinsufficiency in Myod mutant zebrafish. Dev. Biol. 358, 102-112.

Hirai, H., Verma, M., Watanabe, S., Tastad, C., Asakura, Y. and Asakura, A. (2010). MyoD regulates apoptosis of myoblasts through microRNA-mediated down-regulation of Pax3. J. Cell Biol. 191, 347-365.

Huang, W., Sherman, B. T. and Lempicki, R. A. (2008). Systematic and integrative analysis of large gene lists using DAVID bioinformatics resources. Nat. Protoc. 4, 44 57.

Inose, H., Ochi, H., Kimura, A., Fujita, K., Xu, R., Sato, S., Iwasaki, M., Sunamura, S., Takeuchi, Y., Fukumoto, S. et al. (2009). A microRNA regulatory mechanism of osteoblast differentiation. Proc. Natl. Acad. Sci. USA 106, 20794-20799.

Katagiri, T., Yamaguchi, A., Komaki, M., Abe, E., Takahashi, N., Ikeda, T., Rosen, V., Wozney, J. M., Fujisawa-Sehara, A. and Suda, T. (1994). Bone morphogenetic protein-2 converts the differentiation pathway of $\mathrm{C} 2 \mathrm{C} 12$ myoblasts into the osteoblast lineage. J. Cell Biol. 127, 1755-1766.

Kim, H. K., Lee, Y. S., Sivaprasad, U., Malhotra, A. and Dutta, A. (2006). Musclespecific microRNA miR-206 promotes muscle differentiation. J. Cell Biol. 174, 677-687.

Klochendler-Yeivin, A., Picarsky, E. and Yaniv, M. (2006). Increased DNA damage sensitivity and apoptosis in cells lacking the Snf5/Ini1 subunit of the SWI/SNF chromatin remodeling complex. Mol. Cell. Biol. 26, 2661-2674.

Krek, A., Grün, D., Poy, M. N., Wolf, R., Rosenberg, L., Epstein, E. J., MacMenamin, P., da Piedade, I., Gunsalus, K. C., Stoffel, M. et al. (2005) Combinatorial microRNA target predictions. Nat. Genet. 37, 495-500.

Krützfeldt, J., Poy, M. N. and Stoffel, M. (2006). Strategies to determine the biological function of microRNAs. Nat. Genet. 38, S14-S19.

Lewis, B. P., Burge, C. B. and Bartel, D. P. (2005). Conserved seed pairing, often flanked by adenosines, indicates that thousands of human genes are microRNA targets. Cell 120, 15-20.

Lickert, H., Takeuchi, J. K., Von Both, I., Walls, J. R., McAuliffe, F., Adamson, S. L., Henkelman, R. M., Wrana, J. L., Rossant, J. and Bruneau, B. G. (2004). Baf60c is essential for function of BAF chromatin remodelling complexes in heart development. Nature 432, 107-112.

Lopez-Rodríguez, C., Aramburu, J., Rakeman, A. S. and Rao, A. (1999). NFAT5, constitutively nuclear NFAT protein that does not cooperate with Fos and Jun. Proc. Natl. Acad. Sci. USA 96, 7214-7219.

Mankoo, B. S., Skuntz, S., Harrigan, I., Grigorieva, E., Candia, A., Wright, C. V. Arnheiter, H. and Pachnis, V. (2003). The concerted action of Meox homeobox genes is required upstream of genetic pathways essential for the formation, patterning and differentiation of somites. Development 130, 4655-4664.

Mollard, R., Viville, S., Ward, S. J., Décimo, D., Chambon, P. and Dollé, P. (2000) Tissue-specific expression of retinoic acid receptor isoform transcripts in the mouse embryo. Mech. Dev. 94, 223-232.

Nakajima, N., Takahashi, T., Kitamura, R., Isodono, K., Asada, S., Ueyama, T., Matsubara, H. and Oh, H. (2006). MicroRNA-1 facilitates skeletal myogenic differentiation without affecting osteoblastic and adipogenic differentiation. Biochem. Biophys. Res. Commun. 350, 1006-1012.

O'Connor, R. S., Mills, S. T., Jones, K. A., Ho, S. N. and Pavlath, G. K. (2007). A combinatorial role for NFAT5 in both myoblast migration and differentiation during skeletal muscle myogenesis. J. Cell Sci. 120, 149-159.

Rajewsky, N. (2006). microRNA target predictions in animals. Nat. Genet. 38, S8-S13. 
Ramjaun, A. R., Angers, A., Legendre-Guillemin, V., Tong, X. K. and McPherson, P. S. (2001). Endophilin regulates JNK activation through its interaction with the germinal center kinase-like kinase. J. Biol. Chem. 276, 28913-28919.

Rao, P. K., Kumar, R. M., Farkhondeh, M., Baskerville, S. and Lodish, H. F. (2006). Myogenic factors that regulate expression of muscle-specific microRNAs. Proc. Natl. Acad. Sci. USA 103, 8721-8726.

Rao, P. K., Missiaglia, E., Shields, L., Hyde, G., Yuan, B., Shepherd, C. J., Shipley, J. and Lodish, H. F. (2010). Distinct roles for miR-1 and miR-133a in the proliferation and differentiation of rhabdomyosarcoma cells. FASEB J. 24, 3427-3437.

Rathjen, T., Pais, H., Sweetman, D., Moulton, V., Munsterberg, A. and Dalmay, T. (2009). High throughput sequencing of microRNAs in chicken somites. FEBS Lett. $\mathbf{5 8 3}, 1422-1426$

Reijntjes, S., Stricker, S. and Mankoo, B. S. (2007). A comparative analysis of Meox1 and Meox2 in the developing somites and limbs of the chick embryo. Int. J. Dev. Biol. 51, $753-759$.

Ricotti, L., Taccola, S., Bernardeschi, I., Pensabene, V., Dario, P. and Menciassi, A. (2011). Quantification of growth and differentiation of C2C12 skeletal muscle cells on PSS-PAH-based polyelectrolyte layer-by-layer nanofilms. Biomed. Mater. 6, 031001.

Salucci, S., Battistelli, M., Burattini, S., Squillace, C., Canonico, B., Gobbi, P., Papa, S. and Falcieri, E. (2010). C2C12 myoblast sensitivity to different apoptotic chemical triggers. Micron 41, 966-973.

Shan, S. W., Tang, M. K., Chow, P. H., Maroto, M., Cai, D. Q. and Lee, K. K. (2007). Induction of growth arrest and polycomb gene expression by reversine allows $\mathrm{C} 2 \mathrm{C} 12$ cells to be reprogrammed to various differentiated cell types. Proteomics 7 , 4303-4316.
Sweetman, D., Rathjen, T., Jefferson, M., Wheeler, G., Smith, T. G., Wheeler, G. N., Münsterberg, A. and Dalmay, T. (2006). FGF-4 signaling is involved in mir206 expression in developing somites of chicken embryos. Dev. Dyn. 235, 2185-2191.

Sweetman, D., Goljanek, K., Rathjen, T., Oustanina, S., Braun, T., Dalmay, T. and Münsterberg, A. (2008). Specific requirements of MRFs for the expression of muscle specific microRNAs, miR-1, miR-206 and miR-133. Dev. Biol. 321, 491-499.

Swingler, T. E., Wheeler, G., Carmont, V., Elliott, H. R., Barter, M. J., AbuElmagd, M., Donell, S. T., Boot-Handford, R. P., Hajihosseini, M. K., Münsterberg, A. et al. (2012). The expression and function of microRNAs in chondrogenesis and osteoarthritis. Arthritis Rheum. 64, 1909-1919.

Tuddenham, L., Wheeler, G., Ntounia-Fousara, S., Wahters, J., Hajihosseini, M. K., Clark, I. and Dalmay, T. (2006). The cartilage specific microRNA-140 targets histone deacetylase 4 in mouse cells. FEBS Lett 580, 4214-4217.

van Rooij, E., Liu, N. and Olson, E. N. (2008). MicroRNAs flex their muscles. Trends Genet. 24, 159-166.

Wang, W., Xue, Y., Zhou, S., Kuo, A., Cairns, B. R. and Crabtree, G. R. (1996). Diversity and specialization of mammalian SWI/SNF complexes. Genes Dev. 10, 2117-2130.

Wienholds, E., Kloosterman, W. P., Miska, E., Alvarez-Saavedra, E., Berezikov, E., de Bruijn, E., Horvitz, H. R., Kauppinen, S. and Plasterk, R. H. (2005). MicroRNA expression in zebrafish embryonic development. Science 309, 310-311.

Yamamoto-Mizuma, S., Wang, G. X., Liu, L. L., Schegg, K., Hatton, W. J., Duan, D., Horowitz, T. L., Lamb, F. S. and Hume, J. R. (2004). Altered properties of volume-sensitive osmolyte and anion channels (VSOACs) and membrane protein expression in cardiac and smooth muscle myocytes from Clcn3-/- mice. J. Physiol. 557, 439-456 